\title{
Two Photon Absorption in Nanostructure Wide Band Gap Semiconductor CdS Using Femtosecond Laser
}

\author{
Abdulla M. Suhail, Raied K. Jamal, Hani J. Kbashi \\ Department of Physics, College of Sciences, Baghdad University, Baghdad, Iraq \\ E-mail: \{Abdulla_shl, raiedkamel,hani_saka\}@yahoo.com \\ Received September 11, 2011; revised October 18, 2011; accepted October 29, 2011
}

\begin{abstract}
Strong nonlinear absorption in CdS nanoparticles of dimensions in the range $4-50 \mathrm{~nm}$ when irradiated with a femtosecond pulsed laser at $800 \mathrm{~nm}$ is observed. The CdS nanoparticles sizes were controlled by changing the reaction time of aqueous solutions of thiourea and cadmium chloride. The structure of the prepared CdS nanoparticles were tested and their optical properties were investigated and the nonlinear absorption coefficients were measured using a fully computerized z-scan unit. It is found that the formation of the CdS nanostructuresfor are responsible for the observation of the strong tow photon absorption.
\end{abstract}

Keywords: Cadmium Sulfide, Nanostructure, Multiphoton Absorption

\section{Introduction}

Nanoparticle semiconductor materials with large nonlinear coefficients have attracted much attention in recent years [1-5]. This is interest due to their unique optical properties when their size decreased from bulk to few nanometers. Such unusual properties may have technological applications such as optical switching devices, Q-dot lasers, and there is great potential in biophotonics $[6,7]$. The linear optical properties of the semiconductor nanoparticles depends strongly upon the particle size [8]. The nonlinear optical properties, the absorption and refractive index, were observed in nanoparticles semiconductor CdS material [9-11]. The dynamic of the CdS excited state have been studied by femtosecond laser and by the photoluminescence (PL) analysis [12-14]. In this study, a CdS nanofilm was deposited using a simple and low cost spray pyrolysis technique. A fully computerized Z-Scan setup with femtosecond laser was used to investigate the effect of surface state formation in CdS nanoparticles on the nonlinear dynamics parameters.

\section{Experimental}

The CdS nanofilm was prepared by mixing an $0.1 \mathrm{M}$ aqueous solution of thiourea $\left(\mathrm{NH}_{2} \mathrm{CSNH}_{2}\right)$ and an $0.2 \mathrm{M}$ aqueous solution of Cadmium Chloride $\left(\mathrm{CdCl}_{2}\right)$ as starting solution. They were mixed and dissolved in distilled water in the ratio of 3:2. The solution was mixed thor- oughly and the final solution was sprayed onto a heated substrate kept at a temperature of $300^{\circ} \mathrm{C}$. Spray pyrolysis is a useful alterative to the traditional methods for obtaining cadmium sulfide nanofilms, because of its simplicity, low cost and minimal waste production. The spray pyrolysis process allows the coating of a large surface and it is easy to include in an industrial production line. With spray pyrolysis, the solution is sprayed directly onto the substrate. A stream of nitrogen gas can be used to help the spraying of solution through the nozzle.

In the study, a CdS nanofilm was deposited by the spray pyrolysis technique on a glass substrates. The flow rate of the solution experimentally was $5 \mathrm{ml} / \mathrm{min}$ and the substrate temperature was held constant at $300^{\circ} \mathrm{C}$. The nozzle to substrate distance was $28 \mathrm{~cm}$ and the diameter of the nozzle was $0.8 \mathrm{~mm}$. The number of sprays was 15 . The spraying time was controlled by solenoid valve. The heated substrate was left for $10 \mathrm{~s}$ after each spraying run to give time for the deposited CdS layer to be dry and also to prevent excessive cooling of the substrate. This yielded a uniformly grown CdS film on the substrate. The schematic representation of the spray system is given in Figure 1. When the solution is sprayed the following reaction takes place at the surface of the heated substrate.

$$
\begin{aligned}
& \mathrm{CdCl}_{2}+\left(\mathrm{NH}_{2}\right)_{2} \mathrm{CS}+2 \mathrm{H}_{2} \mathrm{O} \\
& \rightarrow \downarrow \mathrm{CdS}+\uparrow 2 \mathrm{NH}_{4} \mathrm{Cl}+\uparrow \mathrm{CO}_{2}
\end{aligned}
$$

During the chemical reaction gas and water vapor is 


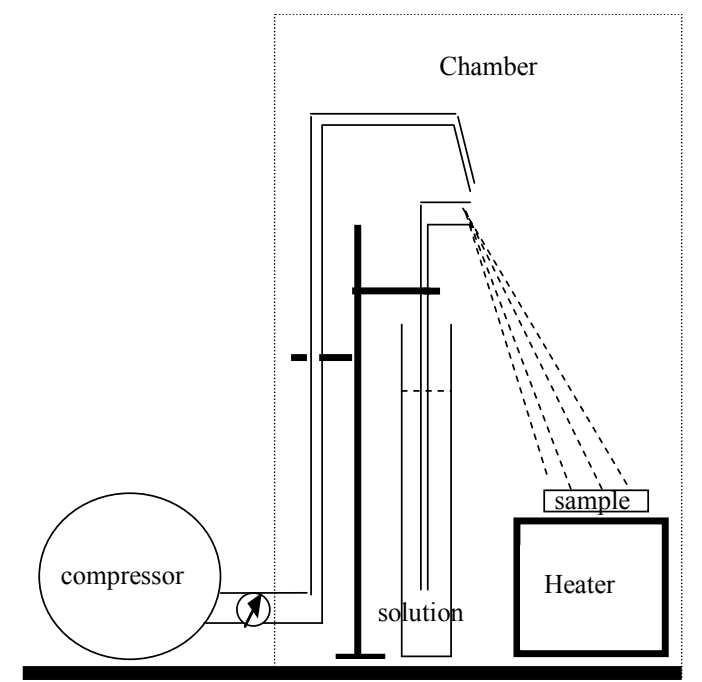

Figure 1. Schematic representation of the spray system.

obtained from this reaction due to the high temperature of the substrate. At the end of the reaction a yellow precipitates remains as a nanofilm of CdS material.

After deposition the film, the material was cooled to room temperature gradually. There are several experimental parameters which are control the homogeneity and the thickness of the nanofilm. These parameters are the spraying time, the height of the atomizer and the pressure of the nitrogen gas carrier. The topography of the prepared nanofilm was studied using Scanning Electron Microscopy (SEM) type ULTRA 55 with different magnifications; as shown in Figure 2. The figures show nanocrystals of size $\sim 4-50 \mathrm{~nm}$. The sample was scanned in all zones before the picture was taken. The micrographs reveal that the particles were hexagonal in shape. The X-ray diffraction (XRD) pattern of the CdS nanofilm was recorded using an XRD 2000 system. The $\mathrm{X}$-ray diffractometer used a copper tube radiation line with a wavelength of $1.54 \AA$ and a $2 \theta$ range from $20^{\circ}$ to $60^{\circ}$. Scan rate was $1 \mathrm{deg} / \mathrm{min}$. The UV-VIS absorption and transmission spectra of the sample were recorded by a Hitachi U-4100 spectrometer covering $200-1100 \mathrm{~nm}$. The photoluminescence (PL) spectrum was studied using an SL1174 spectrophotometer in the range $300-900 \mathrm{~nm}$. The nonlinear absorption study at the near resonant regime was carried out using the single-beam femtosecond open aperture z-scan technique (OA). The z-scan setup is illustrated by the schematic diagram shown in Figure 3. A femtosecond laser with a pulse duration of $51 \mathrm{fs}$ and average power of $351 \mathrm{~mW}$ was used as a laser source. The pulse duration was measured using an autocorrelatior and the energy was measured using a pyroelectric energy probe of model type (PDA36A), covering the wavelength range $350-1100 \mathrm{~nm}$ from THORLABS. The beam profile was adjusted using a spatial filter, leading
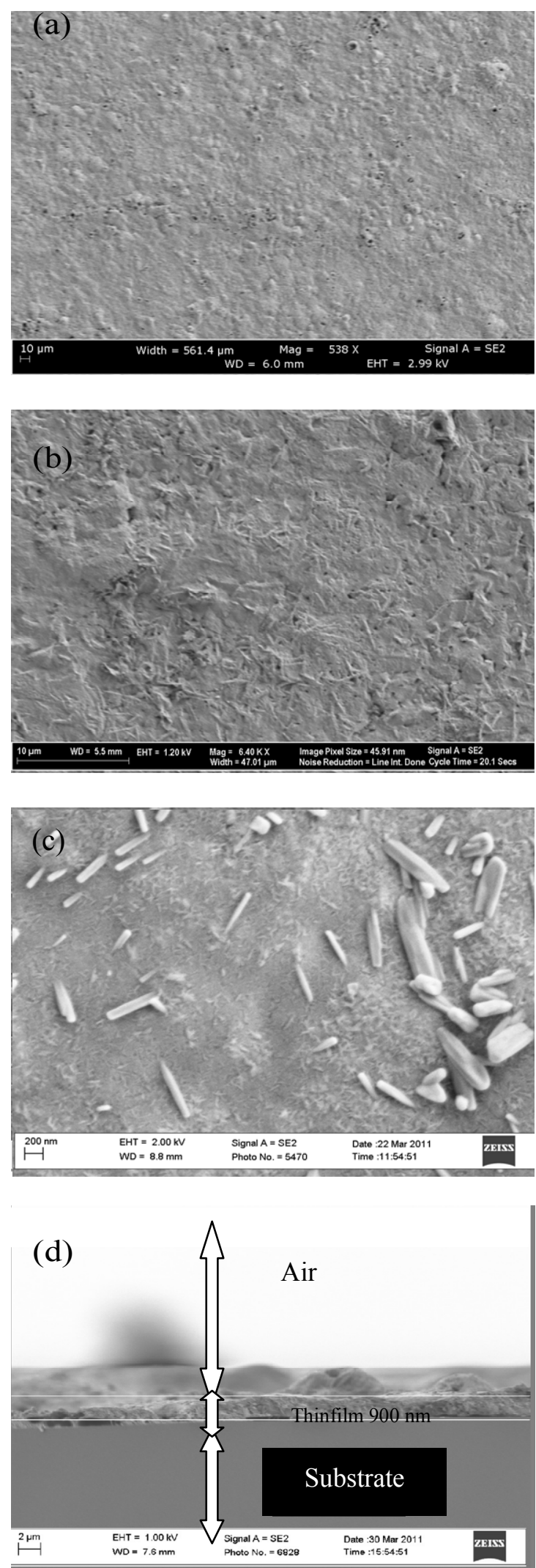

Figure 2. Top-view SEM images of CdS nanofilm at different magnification powers (a)-(c) and cross-section (d). 


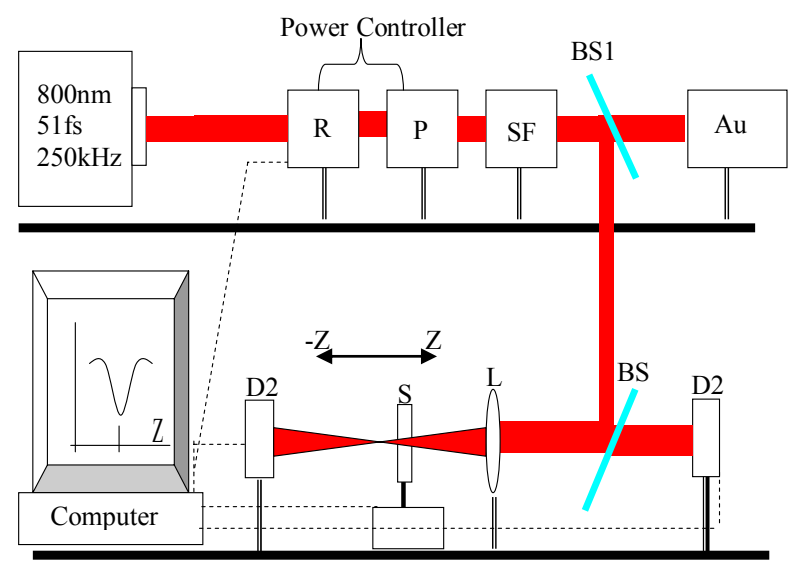

Figure 3. Schematic of the z-scan setup recording the nonlinear absorption, $\mathrm{R}$-Rotator, $\mathrm{P}$-Polarizer, SF-Spatial filter, BS1, BS2-Beam Splitter, Au-Autocorrelation, D1, D2-Detectors, L-Lens, S-Sample.

to spatial intensity profile that was near-Gaussian with beam quality of $\mathrm{M}^{2} \approx 1.36$. The laser beam was focused by a lens with $15 \mathrm{~cm}$ focal length to produce a waist of $32.7 \mu \mathrm{m}$. The sample was translated along the beam axis (z-axis) through the Rayleigh distance $4200 \mu \mathrm{m}$.

\section{Results and Discussion}

The topography study of the prepared film shows the formations of the CdS nano structure and the film thickness was in the range of $0.5-2 \mu \mathrm{m}$. The XRD pattern was recorded for the nanofilm CdS spray-deposited film as shown in Figure 4. The spectrum through $2 \theta=20^{\circ}$ to $2 \theta=60^{\circ}$ indicates that the $\mathrm{CdS}$ nanofilm has a polycrystalline structure. The observed values of the XRD peaks are compared with American Society for Testing and Materials (ASTM) data for hexagonal CdS. The figure shows broad peaks which give evidence of the formation of the nanostructure. Using the width of the (002) peak which appears at an angle of $26.8^{\circ}$ on the $2 \theta$ scale in Scherrer's formula [15]:

$$
d=0.94 \lambda / \beta \cos \theta
$$

where $d$ is the average crystalline grain size, $\lambda$ is the wavelength, $\beta$ represents the full width at half maximum (FWHM) in redian that equal 0.0087 and $\theta$ is the Bragg diffraction angle in degree, the size of the formed nanoparticles was found to be about $50 \mathrm{~nm}$. The absorption spectrum of the CdS nanoparticles film is shown in Figure 5. The film is highly absorbing at wavelength below $500 \mathrm{~nm}$.

The energy band gap of CdS film was estimated using Tauc relation which can be written as [16]:

$$
(\alpha h v)=A(h v-E g) n
$$

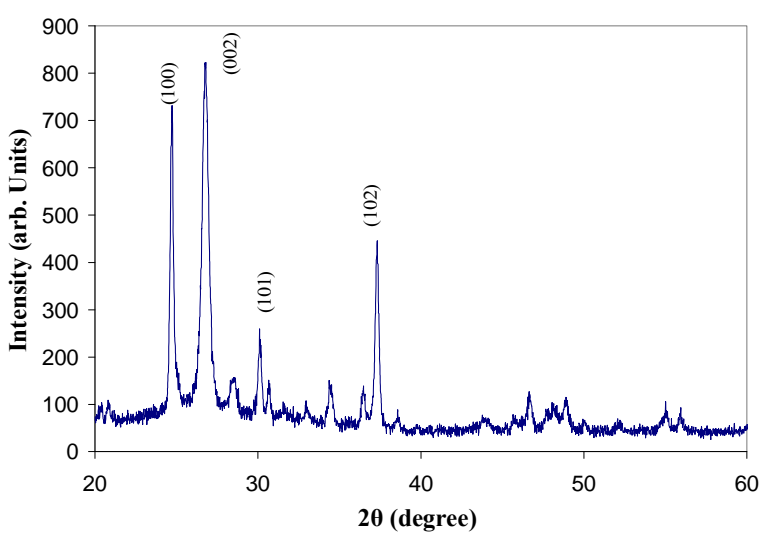

Figure 4. XRD pattern of CdS nanofilm deposited on a glass substrate at $300^{\circ} \mathrm{C}$.

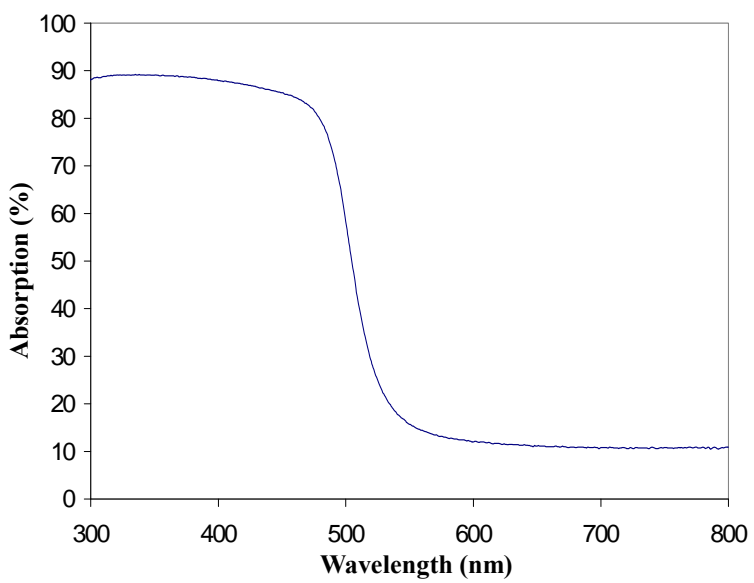

Figure 5. Optical absorption spectra of CdS nanofilm.

where $A$ is a constant, $\alpha$ absorption coefficient, $h v$ the photon energy $(E g)$ the band gap, $n=1 / 2$ for the direct transitions.

Referring to the data extracted from the absorption spectrum, the absorption coefficient was calculated as function of wavelength. Assuming an allowed transition, direct band gap transition, the dependence of $(\alpha h v)^{2}$ on $h v$ is plotted in Figure 6.

The extrapolation of the linear part of the plot to $(\alpha h v)^{2}=0$ gives rise to an estimate the energy gap value of the CdS nanoparticles which was found to be $2.7 \mathrm{eV}$. This value is comparable to the values found by the other workers [17]. The optical transmittance spectrum of the CdS nanofilm is shown in Figure 7.

The transmittance is high in the visible region with a sharp increasing beyond the $520 \mathrm{~nm}$, this indicates that the CdS nanofilm has high absorption below this value because of the occurrence of the linear and nonlinear absorption. The (PL) emission spectrum of CdS nanoparticles excited by a $350 \mathrm{~nm}$ line is shown in Figure 8.

The spectrum shows a peak at $485 \mathrm{~nm}$ which can be re- 


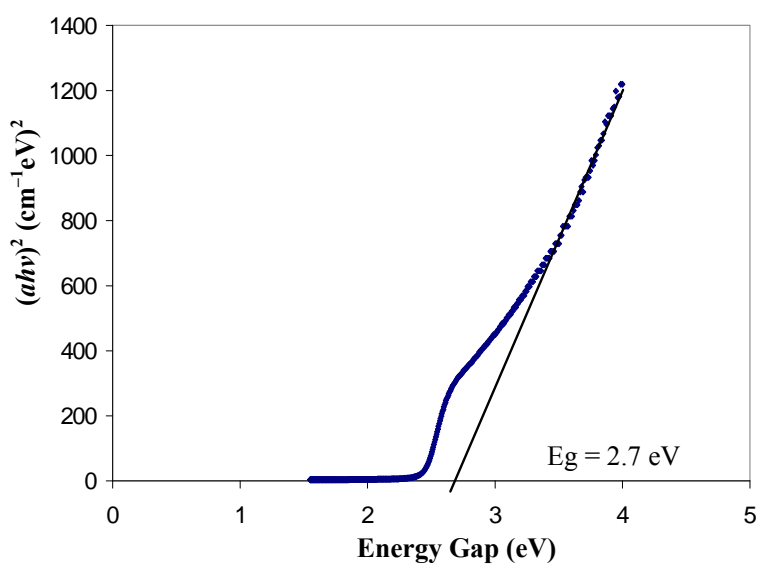

Figure 6. $(\alpha h v)^{2}$ versus enrgy gap.

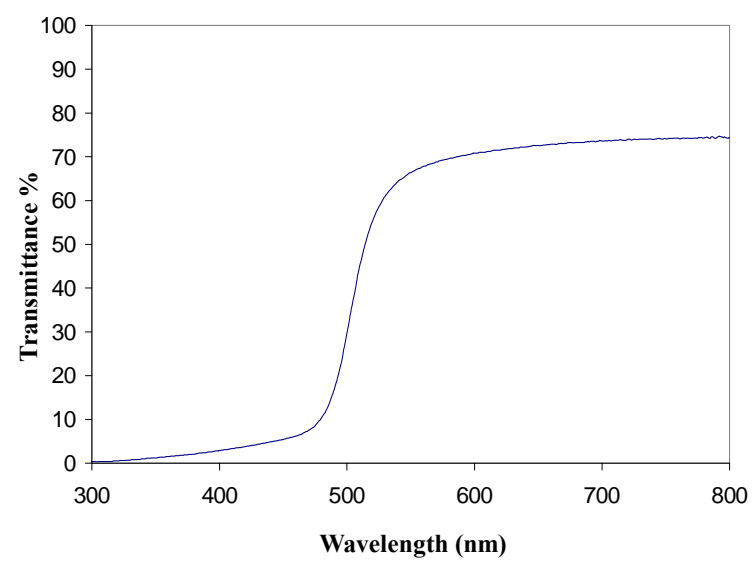

Figure 7. Transmission spectrum of CdS nanofilm.

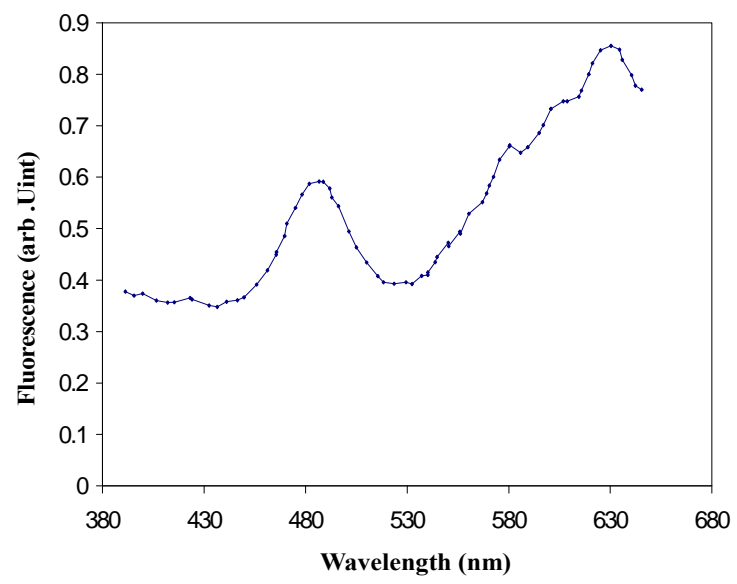

Figure 8. Photoluminescence emission spectrum of CdS nanofilm.

ferred to the direct band gap transition and two peaks around 580 and $630 \mathrm{~nm}$ which are attributed to the formation of surface states. The observation of surface states in the photoluminescence spectrum of the $\mathrm{CdS}$ nanoparticles has been reported by many authors $[18,19]$.
The nanocrystals synthesized with elemental sulfur have more surface defects which act as traps on the nanocrystals surface [20].

The z-scan transition curves for different laser intensities incident on the CdS nanofilm are presented in Figure 9. The normalized transmittance of the open aperture $\mathrm{Z}$-scan is given by [21]:

$$
T(z)=\frac{1}{\pi^{1 / 2} q_{o}} \int_{-\infty}^{\infty} \ln \left[1+q_{o} \exp \left(-x^{2}\right)\right] \mathrm{d} x
$$

where, $q_{o}=\beta I_{o} L_{e f f}, \quad I_{o}=I_{\infty} /\left(1+z^{2} / z_{o}^{2}\right)$ is the excitation intensity at the position $z, z_{o}=\pi \omega z_{o}^{2} / \lambda$ where $z_{o}$ is the Rayleigh range, $\omega_{o}$ is the minimum beam waist at focal point $(z=0), \lambda$ is the laser free-space wavelength, $L_{e f f}=$ $\left[1-\exp \left(-\alpha_{o} L\right)\right] / \alpha_{o}$ is the effective sample length for 2PA processes; $L$ is the sample length and $\alpha_{o}$ is the linear absorption coefficient. The open aperture z-scan graphs are always normalized to linear transmittance i.e., transmittance at large values of $|z|$. The 2PA coefficient can be extracted from the best fit between Equation (3) and the experiment (OA) z-scan curve.

If $q_{o}<1$ Equation (3) can be expanded in a Taylor series as [21]:

$$
T=\sum_{m=0}^{\infty}(-1)^{m} \frac{q_{o}^{m}}{(m+1)^{3 / 2}}
$$

Furthermore, if the higher order terms are ignored, the transmission as a function of the incident intensity is given by $[21,22]$ :

$$
T=1-\frac{\beta I_{o} L_{e f f}}{2^{3 / 2}}
$$

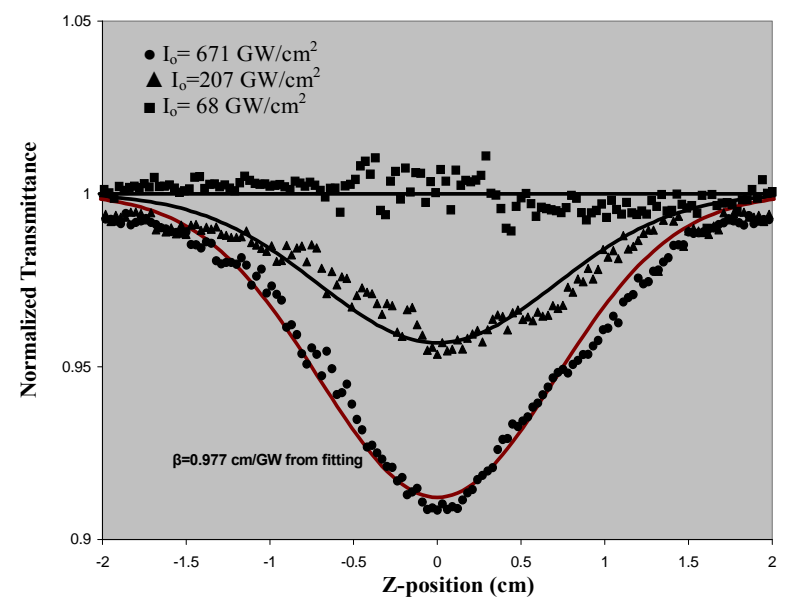

Figure 9. OA z-scan curves measured with different excitation irradiance at a wavelength of $800 \mathrm{~nm}$ and a pulse duration $51 \mathrm{fs}$ and repetition rate of $250 \mathrm{kHz}$. The solid lines are the fitted curves by employing the z-scan theory, described in the text, on 2PA. 
The sold curve in Figure 9 is the best fit for Equation (5). The Equation (5) shows clearly that the depth of the absorption dip is linearly proportional to the 2PA coefficient $\beta$, but the shape of the trace is primarily determined by the Rayleigh range of the focused Gaussian beam. The fitted value of $\beta$ is on the order of $50 \mathrm{~cm} / \mathrm{GW}$. This value is ten times of magnitudes higher than the value observed with bulk CdS sample. This results is in a good agreement with values mentioned in [23]. The natural logarithm of the (1-T) values are plotted as a function of the natural logarithm of the incident intensity $I_{o}$ in Figure 10. The curve can be reasonably fitted with a straight line with a slope of 0.97 . This indicates that the 2PA was occur in CdS pump by $800 \mathrm{~nm}$ laser source of $51 \mathrm{fs}$ pulse duration as shown in Figure 10.

The formation of surface defects may contribute to the absorption mechanism of the prepared film due to small increase in the linear absorption cross section [24]. The formation of the surface defects in CdS and in the other sulfur compound increase the nonlinear scattering leading to decreasing in the nonlinear absorption coefficient. This was observed by viewing the transmittance light through IR camera.

\section{Conclusions}

A CdS nanocrystalline film was prepared by the chemical spray pyrolysis technique. The nonlinear absorption coefficient was measured by fully computerized the zscan technique. The measurements show that the nonlinear absorption coefficient for the nanocrystallites is one order of magnitude higher than that of the bulk CdS material. This increase in the nonlinear responsivity when the crystalline size approaches nano-scale dimensions may be attributed to collimating of the incident intensity of the pumped laser which led to improve the nonlinear

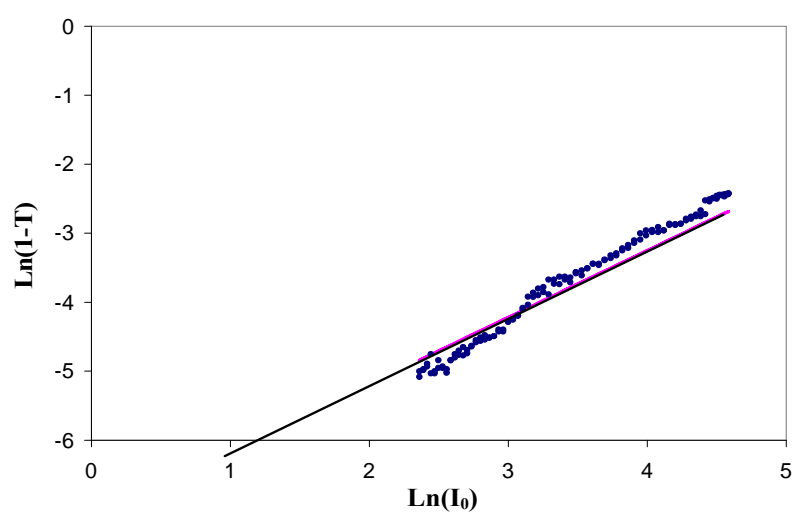

Figure 10. Plot of $\operatorname{Ln}(1-T)$ vs. $\operatorname{Ln}\left(I_{o}\right)$ at $800 \mathrm{~nm}$ wavelength, the solid line is the example of the linear fit at $800 \mathrm{~nm}$ with slope $s=0.97$. dynamic of the CdS nanocrystallite.

\section{Acknowledgements}

This work has been carried out in the physics Department, School of Engineering and Applied Sciences, Harvard University. The authors would like to thanks Mazur Research Group in Harvard University for their help through this work. Thank also to Christopher C. Evans, Jonthan D. B. Bradley, and Eric Mazur for their interest, guide and useful discussion. We thanks also the Ministry of Higher Education in the Republic of Iraq for support this work.

\section{References}

[1] J. H. Bechtel and W. L. Smith, "Two Photon Absorption in Semiconductors with Picosecond Pulses," Physical Review B, Vol. 13, No. 8, 1976, pp. 3515-3518. doi:10.1103/PhysRevB.13.3515

[2] E. W. Van Stryland, H. Vanherzeele, M. A. Woodall, M. J. Soileau, A. L. Smirl, S. Guha and T. F. Boggess, "Two Photon Absorption, Nonlinear Refraction, and Optical Limiting in Semiconductors," Optical Engineering, Vol. 24, 1985, pp. 613-623.

[3] M. Sheik-Bahae, A. A. Said, T. H. Wei, D. J. Hagen and E. W. Van Stryland, "Sensitive Measurement of Optical Nonlinearities Using a Single Beam," IEEE Journal Quantum Electron, Vol. 26, No. 4, 1990, pp. 760-769. doi:10.1109/3.53394

[4] L. W. Tutt and T. F. Boggess, "A Review of Optical Limiting Mechanisms and Devices Using Organics, Fullerenes, Semiconductors and Other Materials," Programming of Quantum Electron, Vol. 17, No. 4, 1993, pp. 299-388. doi:10.1016/0079-6727(93)90004-S

[5] N. Del Fatti and F. Vallce, "Ultrafast Optical Nonlinear Properties of Metal Nanoparticales," Applied Physics, Vol. 73, No. 4, 2001, pp. 383-390.

[6] G. I. Stegman, E. M. Wright, N. Finlayson, R. Zanoni and C. T. Seaton, "Third Order Nonlinear Integrated Optics," IEEE Journal Light Technique, Vol. 6, No. 6, 1988, pp. 953-970. doi:10.1109/50.4087

[7] M. Wegener and W. Schafer, "Semiconductor Optics and Transport Phenomena: From Fundamental to Current Topic," Springer, Berlin, 1997.

[8] P. Nandakumar, C. Vijayan and Y. Murty, "Quantum Size Effects on the Third Order Nonlinearity of CdS Quantum Dots in Nafion," Optical Communication, Vol. 185, No. 4-6, 2000, pp. 457-460. doi:10.1016/S0030-4018(00)01009-9

[9] J. He, W. Ji, G. H. Ma, S. H. Tang, H. I. Elim, W. X. Sun, Z. H. Zhang and W. S. Chin, "Excitonic Nonlinear Absorption in CdS Nanocrystals Studied Using Z-Scan Technique," Journal of Applied Physics, Vol. 95, No. 11, 2004, pp. 6381-6386. doi:10.1063/1.1711183

[10] N. Venkatram and D. N. Rao, "Nonlinear Absorption, 
Scattering and Optical Limiting Studies of CdS Nanoparticles," Optics Express, Vol. 13, No. 3, 2005, pp. 867-871. doi:10.1364/OPEX.13.000867

[11] X. Li, J. V. Embden, J. W. Chon and G. Min, "Enhanced Two-Photon Absorption of CdS Nanocrystal Rods," Applied Physics Letters, Vol. 94, No. 10, 2009, pp. 103117_1103117_3.

[12] V. Klimov, P. H. Bolivar and H. Kurz, "Temperature Study of Trap-Related Photoluminescence Decay in $\mathrm{CdS}_{\mathrm{x}} \mathrm{Se}_{1-\mathrm{x}}$ Nanocrystals in Glass," Physical Review, Vol. B53, 1996, p. 1463.

[13] V. I. Kilmov, Ch. J. Schwarz and D. W. McBranch, “Optical Gain and Stimulate Emission in Nanocrystal Quantum Dots," Physical Review, Vol. B60, 1999, p. 2177.

[14] G. H. Ma, S. H. Tang, W. X. Sun, Z. X. Shen, W. M. Huang and J. L. Shi, "Size-Dependent Excited State Properties of CdS Nanocrystals," Physical Letters, Vol. 299, No. 5-6, 2002, pp. 581-586. doi:10.1016/S0375-9601(02)00680-1

[15] A. L. Patterson, "The Scherrer Formula for X-Ray Particle Size Determination," Physical Review, Vol. 56, No. 10, 1939, pp. 978-982. doi:10.1103/PhysRev.56.978

[16] T. P. Sharma, D. Patidar, N. S. Saxena and K. Sharma, "Measurement of Structural and Optical Band Gaps of $\mathrm{Cd}_{1-\mathrm{x}} \mathrm{Zn}_{\mathrm{x}} \mathrm{S}(\mathrm{x}=4$ and 6) Nanomaterials," Indian Journal of Pure and Applied Physics, Vol. 44, 2006, pp. 125-129.
[17] M. J. Weber, "Hand Book of Optical Materials," 2003.

[18] A. Nag and D. D. Sarma, "White Light from $\mathrm{Mn}^{2+}$-Doped CdS Nanocrystals: A New Approach," Journal Physics Chemistry, Vol. 111, 2007, pp. 13641-13644.

[19] M. D. Garrett, A. D. Dukes and J. R. McBride, "Band Edge Recombination in CdSe, CdS and CdSSe Alloy Nanocrystals Observed by Ultrafast Fluorescence UpConversion: The Effect of Surface Trap States," Journal Physics and Chemistry, Vol. 112, 2008, pp. 12736-12746.

[20] G. G. Yordanov, E. Adachi and C. D. Dushkin, Colloids and Surfaces, Vol. 118, 2006, p. 289.

[21] R. L. Sutherland, D. G. McLean and S. Kirkpatrick, "Handbook of Nonlinear Optics," 2nd Edition, Marcel Dekker, New York, 2003.

[22] T. D. Krauss and F. W. Wise, "Femtosecond Measurement of Nonlinear Absorption and Refraction in CdS, ZnSe, and ZnS," Applied Physics Letter, Vol. 65, No. 14, 1997, p. 1739. doi:10.1063/1.112901

[23] M. Yin, H. P. Li, S. H. Tang and W. Ji, "Determination of Nonlinear Absorption and Reflection by Single Z-Scan Method," Applied Physics, Vol. B70, 2000, pp. 587-591.

[24] J. W. Chon and M. Gu, "Three-Photon Excited Band Edge and Trap Emission of CdS Semiconductor Nanocrystals," Applied Physics Letters, Vol. 84, No. 22, 2004, pp. 4472-4474. doi:10.1063/1.1755420 